\title{
Spatial statistics for environmental studies
}

\author{
Alessandro Fassò • Alessio Pollice . \\ Barbara Cafarelli
}

Received: 14 January 2013 / Accepted: 2 February 2013

(C) Springer-Verlag Berlin Heidelberg 2013

This special issue follows the conference "Spatial Data Methods for Environmental and Ecological Processes-2nd Edition" which started at the University of Foggia, Italy, and continued in the beautiful scenery of Baia delle Zagare on the 1st and 2nd of September 2011. The conference was the 2011 European Regional Conference of The International Environmetrics Society (TIES) and a satellite of the 58th World Statistics Congress of the International Statistical Institute (ISI).

Importantly, the conference was structured on the basis of a largely interdisciplinary project with the aim of creating a space for the exchange of experiences and ideas among researchers from different scientific backgrounds working on spatial and spatiotemporal environmental problems.

The theme of the workshop provided ample space for contributions covering a wide range of territorial, ecological and environmental topics. The Conference's Scientific Committee tailored the program in such a way as to provide the fruitful interaction of various different fields, under the common banner of 'spatial analysis'.

The seven papers of this special issue have a strong motivation on clearly stated environmental problems and consider advanced spatial modeling issues. Three of them are related to advanced spatiotemporal modeling of air quality data at regional scale. Another two papers consider spatial epidemiology, in particular plant infections and malaria. Moreover, one work faces spatial modeling of rainfall extremes at

\footnotetext{
A. Fassò $(\bowtie)$

University of Bergamo, Bergamo, Italy

e-mail: alessandro.fasso@unibg.it

A. Pollice

University of Bari, Bari, Italy

B. Cafarelli

University of Foggia, Foggia, Italy
} 
catchment scale and, last but not least, one is concerned with radioactive contamination of submarine environment.

From the methodological point of view, the seven papers range over non-separable covariances with heuristic estimation, local scoring, non-Gaussian factor models estimated by the stochastic EM algorithm and hierarchical Bayesian modeling approach estimated using either the Monte Carlo Markov Chain algorithm or the recent Integrated Nested Laplace Approximation (INLA) algorithm.

The editors of this special issue thank the referees and the journal editors for making this special issue possible. Their punctual reviews with keen comments and suggestions contributed essentially to the high quality output.

A brief summary of contributions is reported here, following the above application clustering.

Bruno et al. (2012) discuss spatiotemporal hierarchical modeling for heterogenous networks. They consider station heterogeneity by means of station grouping and propose a model class which covers both fixed and random group differences. A model choice technique is also proposed. This approach is applied to tropospheric ozone data in the Emilia-Romagna region, Italy, with monitoring stations classified as background or traffic stations. The results are consistent with known transport effects acting on tropospheric ozone formation.

The paper of Cameletti et al. (2012) reworks a known air quality modeling exercise using the recently introduced approach to Bayesian inference known as INLA. It contains a clear introduction to the approximation of a Gaussian Matérn random field with a Gaussian Markov random field. Moreover, a useful tutorial to the R-library INLA is made while fitting air quality data to a spatiotemporal model characterized by an autoregressive trend with spatially correlated innovations. Comparisons with literature results are given assessing the approximation used.

De Iaco et al. (2012) consider multivariate geostatistical modeling of air borne particulate matters and meteorological variables. While discussing model identification and symmetry issues, they compare two non separable spatiotemporal linear coregionalization models, namely the sum-product variogram and the Gneiting variogram. Prediction capability is assessed for the data from Apulia region, Italy.

Villalta et al. (2012) apply a Bayesian hierarchical log-Poisson model to investigate the spatiotemporal distribution of the incidence of malaria in Sucre state, Venezuela, during the period 1990-2002. They consider several additive regression models for the log-relative risk of disease, including combinations of social-economic and climatic covariates, a spatial random term and a CAR component. Posterior predictive losses and Bayesian $p$-values are used for model comparison and assessment.

Dawid and Musio (2012) utilize the recent discovery that Besag's pseudolikelihood is a special case of a general estimation technique based on proper scoring rules and show that the Brier score can be used with the advantage of being more robust than pseudolikelihood. Thanks to the simple local structure, they apply proper scoring rules to spatial models and illustrate their approach through an example on plant epidemiology concerning phytophthora disease in bell pepper plants.

Bocci et al. (2012) propose a geostatistical model for extreme rainfall, where the observations follow the generalized extreme value distribution with location parameter described by a geoadditive model. Considering its hierarchical structure, the model 
is estimated under the Bayesian paradigm. An application to annual maxima of daily rainfall during the second half of twentieth century in the catchment area of the Arno River in Tuscany, Italy, illustrates the method and calls for the inclusion of a temporal component in the model.

Minozzo and Ferrari (2012), inspired by the military radioactive contamination of the Maddalena Archipelago, Italy, develop a spatial factor model with one factor and bivariate discrete-continuous response. The model parameters are estimated by the maximum likelihood method thanks to a stochastic EM algorithm, while the latent factor is estimated by simulation, namely by Monte Carlo Markov chain approach.

\section{References}

Bocci, C., Caporali, E., Petrucci, A.: Geoadditive modeling for extreme rainfall data. AStA Adv. Stat. Anal. (2012, this issue)

Bruno, F., Cocchi, D., Paci, L.: A practical approach for assessing the effect of grouping in hierarchical spatio-temporal models. AStA Adv. Stat. Anal. (2012, this issue)

Cameletti, M., Lindgren, F., Simpson, D., Rue, H.: Spatio-temporal modeling of particulate matter concentration through the SPDE approach. AStA Adv. Stat. Anal. (2012, this issue)

Dawid, A.P., Musio, M.: Estimation of spatial processes using local scoring rules. AStA Adv. Stat. Anal. (2012, this issue)

De Iaco, S., Palma, M., Posa, D.: Prediction of particle pollution through space-time multivariate analysis AStA Adv. Stat. Anal. (2012, this issue)

Minozzo, M., Ferrari, C.: Multivariate geostatistical mapping of radioactive contamination in the Maddalena Archipelago (Sardinia, Italy). AStA Adv. Stat. Anal. (2012, this issue)

Villalta, D., Guenni, L., Rubio-Palis, Y., Arbelaez, R.: Bayesian space-time modeling of malaria incidence in Sucre state, Venezuela. AStA Adv. Stat. Anal. (2012, this issue) 